Mathematical Research Letters 3, 541-548 (1996)

\title{
RATIONALITY AND EXPONENTIAL GROWTH PROPERTIES OF THE BOUNDARY OPERATORS IN THE NOVIKOV COMPLEX
}

\author{
A. PAjitnov
}

\section{$\S 1$. Introduction}

The classical Morse-Thom-Smale construction associates to a Morse function $g: M \rightarrow \mathbb{R}$ on a closed manifold a chain complex $C_{*}(g)$ of free abelian groups, where the number of free generators of $C_{p}(g)$ equals the number of critical points of $g$ of index $p$ for each $p$. The homology of this complex is isomorphic to the homology of the manifold, and the boundary operator in this complex is defined in a geometric way, using the algebraic number of trajectories of a gradient of $g$, joining critical points of $g$ (see [5], [9], [13], [14], [15]).

In the early 80 s S.P.Novikov generalized this construction to the case of maps $f: M \rightarrow S^{1}$ ( see [6]). Here $M$ is a closed connected manifold, $f: M \rightarrow S^{1}$ is a Morse map, non-homotopic to zero. The corresponding analog of Morse complex is a free chain complex $C_{*}(f)$ over the ring $\mathbb{Z}((t))$ of the formal power series with integer coefficients and finite negative part (that is $\mathbb{Z}((t))=\left\{\sum a_{n} t^{n} \mid a_{n} \in \mathbb{Z}\right.$ and $\exists N: a_{n}=0$ for $\left.n<N\right\}$ ). The number of free generators of $C_{p}(f)$ equals the number of critical points of $f$ of index $p$, and the homology of $C_{*}(f)$ equals to the completed homology of the corresponding cyclic covering of $M$.

The boundary operator in this complex depends on the choice of a Riemannian metric on $M$ or of a gradient-like vector field $v$ for $f$. We prefer in our work the language of gradient-like vector fields (see $\S 2$ for precise definitions). To explicit the boundary operators, let $\bar{M} \stackrel{\mathcal{P}}{\longrightarrow} M$ be the connected infinite cyclic covering for which $f \circ \mathcal{P}$ is homotopic to zero. Choose a lifting $F: \bar{M} \rightarrow \mathbb{R}$ of $f \circ \mathcal{P}$ and let $t$ be the generator of the structure group $(\approx \mathbb{Z})$ of $\mathcal{P}$ such that for every $x \in \bar{M}$ we have $F(x t)<F(x)$. For every critical point $x$ of $f$ choose a lifiting $\bar{x}$ of $x$ to $\bar{M}$. Choose orientations of stable manifolds of critical points. Then for every critical points

Received December 27, 1995. 
$x, y$ of $f$ with ind $x=\operatorname{ind} y+1$, and every $k \in \mathbb{Z}$ the incidence coefficient $n_{k}(x, y ; v)$ is defined as the algebraic number of $(-v)$-trajectories joining $\bar{x}$ to $\bar{y} t^{k}$, each trajectory being counted with the sign, arising from the choice of orientations. (Two trajectories which differ by a choice of parameter, are identified; $v$ is supposed to satisfy the transversality assumption.) Set $n(x, y ; v)=\sum_{k \in \mathbb{Z}} n_{k}(x, y ; v) t^{k} \in \mathbb{Z}((t))$. Now the boundary operator $\partial: C_{m}(f) \rightarrow C_{m-1}(f)$ is defined by $\partial x=\sum_{y} y \cdot n(x, y ; v)$, where $x$ is a critical point of $f$ of index $m$, and the sum ranges over critical points of $f$ of index $m-1$.

Since the beginning S. P. Novikov conjectured that the power series $n(x, y ; v)$ had some nice analytic properties. In particular he conjectured that

$$
\begin{gathered}
(\mathcal{E}) \text { Generically the coefficients } n_{k}(x, y ; v) \\
\text { grow at most exponentially with } k .
\end{gathered}
$$

This conjecture is stated in [7] for the case of analytic Morse maps $f$. The present paper announces that generically the coefficients $n(x, y ; v)$ are rational functions in $t$, which implies the exponential estimate (see $\S 2$ for precise statement).

Further, denote by $N_{k}(x, y ; v)$ the total number of $(-v)$-trajectories joining $\bar{x}$ to $\bar{y} t^{k}$. The version of the conjecture $(\mathcal{E})$ presented in $[1, \mathrm{p}$. 83] states that generically the numbers $N_{k}(x, y ; v)$ grow at most exponentially with $k$. (There is also the corresponding statement for Morse forms.) Closely related to it is the question of $\mathrm{V}$. I. Arnold about the asymptotic behaviour of $A^{n} X \cap Y$ where $A$ is a diffeomorphism of a manifold $M$ to itself, $X, Y$ are submanifolds of $M$ and $n \rightarrow \infty$ (see [1,2]).

We confirm the exponential estimate for $N_{k}(x, y ; v)$ as well; see $\S 4$ for the precise statement as well as for the statement of the corresponding result for Morse forms.

Using the universal covering $\widetilde{M} \rightarrow M$ instead of the cyclic covering above, one obtains a version of Novikov complex defined over corresponding completion of the group ring $\mathbb{Z} \pi_{1} M$. We announce in $\S 3$ that the corresponding incidence coefficients belong to the (non commutative) localization in the sense of P. M. Cohn of the ring $\mathbb{Z} \pi_{1} M$.

Instead of Morse maps $M \rightarrow S^{1}$ one can consider Morse 1-forms (recall that Morse form is a closed 1-form, which is locally a differential of a Morse function). To each Morse form $\omega$ one associates the homomorphism of integration $H(\omega): H_{1}(M, \mathbb{Z}) \rightarrow \mathbb{R}$ and the free $\mathbb{Z}^{k}$-covering $M^{\prime} \rightarrow M$ corresponding to the kernel of the composition $\pi_{1} M \rightarrow H_{1}(M, \mathbb{Z}) \stackrel{H(\omega)}{\longrightarrow} \mathbb{R}$ 
(where $k=\mathrm{rk} \operatorname{Im} H(\omega)$ ). The corresponding incidence coefficients belong to a suitable completion of the Laurent polynomial ring $\mathbb{Z}\left[\mathbb{Z}^{k}\right]$. We announce that generically they belong to the corresponding localization of $\mathbb{Z}\left[\mathbb{Z}^{k}\right]$ (see Theorem 3 of the present paper, which states a bit stronger assertion, concerning the maximal free abelian coverings).

The full proof of Theorem 1 is contained in [11]. The full proofs of the results of $\S 3,4$ are contained in [12].

\section{$\S$ 2. Incidence coefficients with values in $\mathbb{Z}((t))$}

We need some definitions. Let $f: M \rightarrow \mathbb{R}^{1}$ be a Morse function on a manifold $M, \operatorname{dim} M=n$. The set of critical points of $f$ will be denoted by $S(f)$. Let $v$ be a vector field on $M$ (we assume that all objects are of class $\left.C^{\infty}\right)$. We say that $v$ is an $f$-gradient if for every non-critical point $x$ of $f$ we have $d f(v)(x)>0$ and for every critical point $p$ of $f$ there is a chart $\Phi: W \rightarrow V$, where $W$ is an open neighborhood of $p$, and $V$ is an open neighborhood of 0 in $\mathbb{R}^{n}$, such that $\Phi(p)=0$ and

(1) $\left(f \circ \Phi^{-1}\right)\left(x_{1}, \ldots, x_{n}\right)=f(p)+\sum_{i=1}^{n} \alpha_{i} x_{i}^{2}$ with $\alpha_{m}<0$ for $m \leqslant k$ and $\alpha_{m}>0$ for $m>k$.

(2) $\Phi_{*}(v)=\left(-x_{1}, \ldots,-x_{k}, x_{k+1}, \ldots, x_{n}\right)$.

(here $k$ is the index of the critical point $p$ ). The set of all $f$-gradients will be denoted by $\mathcal{G}(f)$, and the set of all $f$-gradients satisfying the transversality assumption will be denoted by $\mathcal{G} t(f)$. We assume similar terminology for maps $f: M \rightarrow S^{1}$.

Theorem 1. Let $M$ be a closed connected manifold, $f: M \rightarrow S^{1}$ be a Morse map, non homotopic to zero. Then in the set $\mathcal{G} t(f)$ there is a subset $\mathcal{G} t_{0}(f)$ with the following properties:

(1) $\mathcal{G} t_{0}(f)$ is open and dense in $\mathcal{G} t(f)$ with respect to $C^{0}$ topology.

(2) If $v \in \mathcal{G} t_{0}(f), x, y \in S(f)$ and ind $x=\operatorname{ind} y+1$, then $\sum_{k \in \mathbb{Z}} n_{k}(x, y ; v) t^{k}$ is a rational function of $t$ of the form $\frac{P(t)}{t^{m} Q(t)}$, where $P(t)$ and $Q(t)$ are polynomials with integral coefficients, $m \in \mathbf{N}$, and $Q(0)=1$.

(3) Let $v \in \mathcal{G} t_{0}(f)$. Let $U$ be a neighborhood of $S(f)$. Then for every $w \in \mathcal{G} t_{0}(f)$ such that $w=v$ in $U$ and $w$ is sufficiently close to $v$ in $C^{0}$ topology we have: $n_{k}(x, y ; v)=n_{k}(x, y ; w)$ for every $x, y \in S(f)$ with ind $x=$ ind $y+1$, and every $k \in \mathbb{Z}$.

Remarks.

1)The exponential estimate in $(\mathcal{E})$ follow immediately, since the the Taylor series of every rational function of the form $\frac{P(t)}{Q(t)}$ with $Q(t) \neq 0$ has a 
non-zero radius of convergency.

2) From the main theorem of [10] it follows that for every finite sequence $a_{1}, \ldots, a_{k}$ of integers there is a Morse map $f: M \rightarrow S^{1}$ on a manifold $M$, an $f$-gradient $v$ and critical points $x, y$ of $f$ with ind $x=\operatorname{ind} y+1$ such that $n_{0}(x, y ; v)=1$ and $n_{i}(x, y ; v)=a_{i}$ for $1 \leqslant i \leqslant k$.

\section{$\S 3$. Incidence coefficients with values in completions of group rings}

To state the results we recall some algebraic and Morse-theoretic definitions.

Let $G$ be a group and $\xi: G \rightarrow \mathbb{R}$ be a group homomorphism. We denote by $(\mathbb{Z} G)^{\wedge}$ the abelian group of all formal linear combinations $\sum_{g \in G} n_{g} g$, infinite in general (where $n_{g} \in \mathbb{Z}$ ). Novikov ring $\mathbb{Z} G_{\xi}^{-}$is the ring of such $\lambda \in(\mathbb{Z} G)^{\wedge}$, that for every $c \in \mathbb{R}$ the set supp $\lambda \cap \xi^{-1}([c, \infty[)$ is finite.

Let $\omega$ be a closed 1 -form on a manifold $M$. The deRham cohomology class of $\omega$ will be denoted by $[\omega]$ and the corresponding homomorphism $\pi_{1} M \rightarrow \mathbb{R}$ will be denoted by $\{\omega\}$. We say that $\omega$ is a Morse form, if locally it is the differential of a Morse function. The set of zeros of $\omega$ will be denoted by $S(\omega)$. A Morse form $\omega$ is the differential of a Morse map $f: M \rightarrow S^{1}$ if and only if $[\omega] \in \operatorname{Im}\left(H^{1}(M, \mathbb{Z}) \rightarrow H^{1}(M, \mathbb{R})\right)$.

An obvious generalization of the definition of $f$-gradient for Morse maps to $S^{1}$ gives the notion of $\omega$-gradient of a Morse form $\omega$. The set of all $\omega$ gradients will be denoted by $\mathcal{G}(\omega)$ and the set of all $\omega$-gradients satisfying the transversality assumption will be denoted by $\mathcal{G} t(\omega)$. Let $\omega$ be a Morse form on a closed connected manifold $M$ and let $v \in \mathcal{G} t(\omega)$. For each zero $x$ of $\omega$ choose a lifting $\widetilde{x}$ of $x$ to $\widetilde{M}$ and an orientation of the stable manifold of $x$. Then for every $x, y \in S(\omega)$ with ind $x=\operatorname{ind} y+1$ the incidence coefficient $\widetilde{n}(\widetilde{x}, \widetilde{y} ; v) \in\left(\mathbb{Z} \pi_{1} M\right)_{\xi}^{-}$can be defined (the definition is similar to that of $n(x, y ; v)$ in $\S 1$; for the case of Morse maps $M \rightarrow S^{1}$ one can find it in [9].)

(Although we shall not use the notion of Novikov complex in this paper, we give the definition. Let $C_{p}(\omega)$ be the free right $\mathbb{Z}\left(\pi_{1} M\right)_{\xi}^{-}$-module, generated by $S_{p}(\omega)$, where $S_{p}(\omega)$ stands for the set of zeros of $\omega$ of index $p$. Define a homomorphism $\partial_{p}: C_{p}(\omega) \rightarrow C_{p-1}(\omega)$ by $\partial_{p} x=\sum_{y \in S_{p-1}(\omega)} y \cdot \widetilde{n}(\widetilde{x}, \widetilde{y} ; v)$, where $x \in S_{p}(\omega)$. One can prove that $\partial_{p} \circ \partial_{p+1}=0$; the resulting complex is called Novikov complex .)

1. Morse maps $M \rightarrow S^{1}$

Let $\xi: G \rightarrow \mathbb{Z}$ be a group epimorphism. Denote Ker $\xi$ by $H$. For $n \in \mathbb{Z}$ denote $\xi^{-1}(n)$ by $G_{(n)}$ and $\left\{x \in \mathbb{Z} G \mid \operatorname{supp} x \subset G_{(n)}\right\}$ by $\mathbb{Z} G_{(n)}$. Denote 
$\left.\left.\xi^{-1}(]-\infty,-1\right]\right)$ by $G_{-}$and $\left\{x \in \mathbb{Z} G \mid \operatorname{supp} x \subset G_{-}\right\}$by $\mathbb{Z} G_{-}$. Choose $\theta \in \mathbb{Z} G_{(-1)}$. It is easy to see that $(\mathbb{Z} G)_{\xi}^{-}$is identified with the ring of power series of the form $\sum_{i=-\infty}^{\infty} a_{i} \theta^{i}$, where $a_{i} \in \mathbb{Z} H$ and the negative part of the series is finite.

Set $\Sigma_{n}=\left\{\mathbf{1}+A \mid A \in \operatorname{Mat}_{n \times n}\left(\mathbb{Z} G_{(-1)}\right)\right\}$. Set $\Sigma=\bigcup_{n \geqslant 1} \Sigma_{n}$.

There is the corresponding localization ring $\mathbb{Z} G_{\Sigma}$ (see [4, p.255]). Every matrix in $\Sigma_{n}$ is invertible in $\operatorname{Mat}_{n \times n}\left(\mathbb{Z} G_{\xi}^{-}\right)$, the inverse of $\mathbf{1}+A$ being given by $\sum_{n=0}^{\infty}(-1)^{n} A^{n}$, therefore the localization map $\lambda: \mathbb{Z} G \rightarrow \mathbb{Z} G_{\Sigma}$ is injective and the inclusion $i: \mathbb{Z} G \hookrightarrow \mathbb{Z} G_{\xi}^{-}$factors through a ring homomorphism $\ell: \mathbb{Z} G_{\Sigma} \rightarrow \mathbb{Z} G_{\xi}^{-}$.

Proceeding to the statement of the next result, let $M$ be a connected closed manifold and $f: M \rightarrow S^{1}$ be a Morse map, nonhomotopic to zero. Denote by $\xi$ the induced homomorphism $\pi_{1} M \rightarrow \mathbb{Z}$. Assume that $\xi$ is epimorphic. Then by the previous discussion we have the localization $\left(\mathbb{Z} \pi_{1} M\right)_{\Sigma}$ and the homomorphism $\ell:\left(\mathbb{Z} \pi_{1} M\right)_{\Sigma} \rightarrow\left(\mathbb{Z} \pi_{1} M\right)_{\xi}^{-}$.

Theorem 2. In $\mathcal{G} t(f)$ there is a subset $\mathcal{G} t_{1}(f)$ with the following properties:

(1) $\mathcal{G} t_{1}(f)$ is open and dense in $\mathcal{G} t(f)$ with respect to $C^{0}$ topology.

(2) If $v \in \mathcal{G} t_{1}(f)$ then for every $x, y \in S(f)$ with ind $x=\operatorname{ind} y+1$ we have $\widetilde{n}(\widetilde{x}, \widetilde{y} ; v) \in \operatorname{Im} \ell$.

(3) Let $v \in \mathcal{G} t_{1}(f)$. Let $U$ be a neighborhood of $S(f)$. Then for every $w \in \mathcal{G} t_{1}(f)$ such that $w=v$ in $U$ and $w$ is sufficiently close to $v$ in $C^{0}$ topology we have: $\widetilde{n}(\widetilde{x}, \widetilde{y} ; v)=\widetilde{n}(\widetilde{x}, \widetilde{y} ; w)$ for every $x, y \in S(f)$ with ind $x=$ ind $y+1$.

\section{Morse forms within arbitrary cohomology classes}

Let $\omega$ be a Morse form on a closed connected manifold $M$ and let $\phi$ : $\overline{\bar{M}} \rightarrow M$ be any connected regular covering with structure group $G$ such that $\phi^{*}([\omega])=0$. Then the homomorphism $\{\omega\}: \pi_{1} M \rightarrow \mathbb{R}$ factors as $\pi_{1} M \rightarrow G \stackrel{\langle\omega\rangle}{\longrightarrow} \mathbb{R}$. Let $v \in \mathcal{G} t(\omega)$. For every $x \in S(\omega)$ choose a lifting $\overline{\bar{x}}$ of $x$ to $\overline{\bar{M}}$ and an orientation of the stable manifold of $x$. Then for every $x, y \in S(\omega)$ with ind $x=\operatorname{ind} y+1$ the incidence coefficient $\overline{\bar{n}}(\overline{\bar{x}}, \overline{\bar{y}} ; v) \in \mathbb{Z} G_{\langle\omega\rangle}^{-}$ is defined (similarly to $\widetilde{n}(\widetilde{x}, \widetilde{y} ; v)$ ).

In particular it is the case for the maximal free abelian covering $\widehat{M} \stackrel{\mathcal{P}}{\longrightarrow} M$ with the structure group $H_{1}(M, \mathbb{Z}) /$ Tors $\approx \mathbb{Z}^{m}$. The corresponding homomorphism $\mathbb{Z}^{m} \rightarrow \mathbb{R}$ is the one arising from the de Rham cohomology class 
$[\omega]$; it will be denoted by the same symbol $[\omega]$. Set $S_{[\omega]}=\left\{P \in \mathbb{Z}\left[\mathbb{Z}^{m}\right] \mid P=\right.$ $1+Q$ and $\left.\operatorname{supp} Q \subset[\omega]^{-1}(]-\infty, 0[)\right\}$.

Theorem 3. Let $\omega$ be a Morse form with $[\omega] \neq 0$. Then there is a subset $\mathcal{G} t_{1}(\omega) \subset \mathcal{G} t(\omega)$ with the following properties:

(1) $\mathcal{G} t_{1}(\omega)$ is open and dense in $\mathcal{G} t(\omega)$ with respect to $C^{0}$ topology.

(2) For every $v \in \mathcal{G} t_{1}(\omega)$ and every $x, y \in S(\omega)$ with ind $x=\operatorname{ind} y+1$ we have: $\widehat{n}(\widehat{x}, \widehat{y} ; v) \in S_{[\omega]}^{-1} \mathbb{Z}\left[\mathbb{Z}^{m}\right]$.

(3) Let $v \in \mathcal{G} t_{1}(\omega)$. Let $U$ be a neighborhood of $S(\omega)$. Then for every $w \in \mathcal{G t}_{1}(\omega)$ such that $w=v$ in $U$ and $w$ is sufficiently close to $v$ in $C^{0}$ topology we have: $\widehat{n}(\widehat{x}, \widehat{y} ; v)=\widehat{n}(\widehat{x}, \widehat{y} ; w)$ for every $x, y \in S(\omega)$ with ind $x=$ ind $y+1$.

\section{Exponential growth estimates}

Let $G$ be a group. For an element $a=\sum n_{g} g \in \mathbb{Z} G$ we denote by $\|a\|$ the sum $\sum\left|n_{g}\right|$.

Let $\xi: G \rightarrow \mathbb{R}$ be a homomorphism . For $\lambda=\sum_{g} n_{g} g \in \mathbb{Z} G_{\xi}^{-}$and $c \in \mathbb{R}$ we denote by $\lambda[c]$ the element $\sum_{\xi(g) \geqslant c} n_{g} g$ of $\mathbb{Z}\left[\pi_{1} M\right]$ and we set $N_{c}(\lambda)=\|\lambda[c]\|$. We shall say that $\lambda$ is of exponential growth if there are $A, B \geqslant 0$ such that for every $c \in \mathbb{R}$ we have $N_{c}(\lambda) \leqslant A e^{-c B}$. It is easy to prove that the elements of exponential growth form a subring of $\mathbb{Z} G_{\xi}^{-}$, which contains $\mathbb{Z} G$.

Theorem 4. Let $\omega$ be a Morse form with $[\omega] \neq 0$, and let $v$ be an $\omega$ gradient, belonging to $\mathcal{G} t_{1}(\omega)$. Let $x, y \in S(\omega)$, ind $x=\operatorname{ind} y+1$. Then $\widetilde{n}(\widetilde{x}, \widetilde{y} ; v)$ is of exponential growth .

\section{$\S 4$. Exponential growth estimate of the total number of trajectories}

1. Morse maps $M \rightarrow S^{1}$

We assume here the terminology of $\S 1$. Recall from $[10, \S 2 \mathrm{~B}]$ that an $f$-gradient $v$ is called good if for every $p, q \in S(f)$ we have

$$
(\operatorname{ind} p \leqslant \operatorname{ind} q+1) \Rightarrow(D(p, v) \pitchfork D(q,-v)) .
$$

The set of all good $f$-gradients will be denoted by $\mathcal{G} d(f)$. For $p, q \in S(F)$ with ind $p=\operatorname{ind} q+1$ and for $v \in \mathcal{G} d(f)$ the set of $(-v)$ - trajectories joining 
$p$ to $q$ is finite. For $x, y \in S(f)$ with ind $x=\operatorname{ind} y+1$ and $k \in \mathbb{Z}$ denote by $N_{k}(x, y ; v)$ the number of $(-v)$ - trajectories joining $\bar{x}$ to $\bar{y} t^{k}$ (recall that $\bar{x}, \bar{y}$ stand for the chosen liftings of $x, y$ to $\bar{M})$.

Theorem 5. In the set $\mathcal{G}(f)$ there is a subset $\mathcal{G}_{0}(f)$ with the following properties:

(1) $\mathcal{G}_{0}(f)$ is $C^{0}$ dense in $\mathcal{G}(f)$ and $\mathcal{G}_{0}(f) \subset \mathcal{G} d(f)$.

(2) Let $v \in \mathcal{G}_{0}(f)$. Then there are constants $C, D>0$ such that for every $x, y \in S(f)$ with ind $x=\operatorname{ind} y+1$ and for every $k \in \mathbb{Z}$ we have $N_{k}(x, y ; v) \leqslant C \cdot D^{k}$.

\section{Morse forms within arbitrary cohomology classes}

We assume here the terminology of $\S 3$. Further, for a Morse form $\omega$ on $M$ we denote by $\mathcal{G} d(\omega)$ the set of all the good $\omega$-gradients . (The definition of a good $\omega$-gradient is similar to that of good $f$-gradient from the above.)

For any $v \in \mathcal{G} d(\omega)$, any $x, y \in S(\omega)$ with ind $x=$ ind $y+1$ and any $g \in \pi_{1} M$ the set of $(-v)$ - trajectories joining $\widetilde{x}$ to $\widetilde{y} g$ is finite and we denote its cardinality by $N(\widetilde{x}, \widetilde{y}, g ; v)$. For $c \in \mathbb{R}$ we denote by $N_{\geqslant c}(\widetilde{x}, \widetilde{y} ; v)$ the sum $\sum_{g:\{\omega\}(g) \geqslant c} N(\widetilde{x}, \widetilde{y}, g ; v)$.

Theorem 6. In the set $\mathcal{G}(\omega)$ there is a subset $\mathcal{G}_{0}(\omega)$ with the following properties:

(1) $\mathcal{G}_{0}(\omega)$ is dense in $\mathcal{G}(\omega)$ with respect to $C^{0}$ topology; $\mathcal{G}_{0}(\omega) \subset \mathcal{G} d(\omega)$.

(2) Let $v \in \mathcal{G}_{0}(\omega)$. There are constants $C, D>0$ such that for every $x, y \in S(\omega)$ with ind $x=\operatorname{ind} y+1$ and every $\lambda \in \mathbb{R}$ we have $N_{\geqslant \lambda}(x, y ; v) \leqslant C \cdot D^{-\lambda}$.

\section{References}

1. V. Arnold, Dynamics of intersections, Proc. Conf. in Honour of J. Moser (P. Rabinowitz and R. Hill, eds.), Academic Press, 1990, pp. 77-84.

2. _ Dynamics of complexity of intersections, Bol. Soc. Brasil. Mat. (1990), 1 10.

3. N. Bourbaki, Algèbre commutative, Masson, 1985.

4. P. M. Cohn, Free rings and their relations, Academic press, 1971.

5. M. Morse, Calculus of variations in the large, Amer. Math. Soc. Colloq. Publ. Vol.18, 1934.

6. S. P. Novikov, Mnogoznachnye funktsii i funktsionaly. Analog teorii Morsa, Dokl. Akad. Nauk SSSR 260 (1981), 31-35 (Russian); English translation: Many-valued functions and functionals. An analogue of Morse theory., Sov. Math. Dokl. 24 (1981), 222-226.

7. _ Quasiperiodic structures in topology, Topol. Methods Modern Math., Publish or Perish, Inc, 1993, pp. $223-235$. 
8. A. V. Pajitnov, On modules over some localizations of Laurent polynomial rings, Math. Notes Akad. Nauk SSSR 46 (1989), no. 5, 40 - 49. (Russian)

9. , On the Novikov complex for rational Morse forms, Ann. Fac. Sci. Toulouse Math. 4 (1995), 297 - 338.

10. Surgery on the Novikov complex, K-theory 10 (1996).

11. __ The incidence coefficients in the Novikov complex are generically rational functions; e-print dg-ga/ 9603006 (14 Mar 1996).

12. Incidence coefficients in the Novikov complex for Morse forms: rationality and exponential growth properties; e-print dg-ga/ 9604004 (20 Apr 1996).

13. S. Smale, On the structure of manifolds, Amer. J. Math. 84 (1962), 387-399.

14. R. Thom, Sur une partition en cellules associée à une fonction sur une variété, C. R. Acad. Sci. 228 (1949), 973-975.

15. E. Witten, Supersymmetry and Morse theory, J. Diff. Geom. 17 (1985).

Univerité De Nantes, Département De Mathématiques, 2 Rue De La H OUSSINIÈre, 44072 N ANTES CEDEX

E-mail address: pajitnov@univ-rennes1.fr 